\title{
Escaping the repugnant conclusion: Rank-discounted utilitarianism with variable population
}

\author{
Geir B. Asheim \\ Department of Economics, University of Oslo
}

STÉPHANE ZuBER

Paris School of Economics and CNRS, Centre d'Économie de la Sorbonne

\begin{abstract}
We contribute to population ethics by proposing and axiomatizing rankdiscounted critical-level generalized utilitarianism (RDCLU). Population ethics is needed for evaluation of policies, e.g., concerning climate change, where population size depends on the chosen policy. We show that critical-level generalized utilitarianism and (a version of) critical-level leximin are the limits of RDCLU for extreme values of the rank utility discount factor. Moreover, we establish how RDCLU avoids serious objections raised against other variable population criteria. In particular, it escapes both the Repugnant Conclusion and the Very Sadistic Conclusion (while critical-level generalized utilitarianism leads to one of these undesirable conclusions for any critical level).
\end{abstract}

KEYwords. Social evaluation, population ethics, critical-level utilitarianism, social discounting.

JEL CLAssification. D63, D71, H43, Q56.

\section{INTRODUCTION}

Emission of greenhouse gases may prevent the existence of a great many people who would otherwise have existed, as the earth will not be able to sustain anything approaching our present population if there is extreme climate change (Broome 2010). How should we take into account the loss of such potential lives when evaluating policies designed to abate greenhouse gas emissions?

Practical evaluation of climate polices in integrated assessment models, like versions of Nordhaus' Dynamic Integrated model of Climate and the Economy (DICE model,

Geir B. Asheim: g.b.asheim@econ.uio.no

Stéphane Zuber: stephane .zuber@univ-paris1.fr

We thank an associate editor and two referees for detailed and constructive comments, Gustaf Arrhenius, Charles Figuières, and Reyer Gerlagh for helpful discussions, and seminar and conference participants in Marseille, New Delhi, Paris, and Seoul for valuable suggestions. This paper is part of the research activities at the Centre for the Study of Equality, Social Organization, and Performance (ESOP) at the Department of Economics at the University of Oslo. ESOP is supported by the Research Council of Norway. Asheim's research has also been supported by l'Institut d'Études Avancées-Paris. Zuber's research has been supported by the Chair on Welfare Economics and Social Justice at the Institute for Global Studies (FMSH-Paris) and the Franco-Swedish Program on Economics and Philosophy (FMSH and Riksbankens Jubileumsfond).

Copyright @ 2014 Geir B. Asheim and Stéphane Zuber. Licensed under the Creative Commons AttributionNonCommercial License 3.0. Available at http: //econtheory . org. 
Nordhaus 2008), usually applies the time-discounted utilitarian criterion, implying that insignificant weight is assigned to people living centuries from now. Since serious climate change will, in particular, reduce population size in the far future, accounting for loss of potential lives is not of great concern under time-discounted utilitarianism.

However, if one instead denounces such deviation from the ideal of equal treatment of all individuals, then the issue of losing potential lives, even many generations from now, becomes much more pressing. If we endorse Broome's (2004) position that one cannot simply ignore the effects on population size when evaluating climate policies, while maintaining that all individuals be treated equally, what kind of evaluation criterion should we use?

One prominent response is to assume that there exists a critical level of lifetime wellbeing which, if experienced by an added individual without changing the well-being levels of the existing population, leads to an alternative that is as good as the original. This critical level may differ from the (individually) neutral level of well-being, above which a life is worth living and below which it is not. Combined with a generalized utilitarian criterion where, for fixed population size, the undiscounted sum of the individuals' (transformed) utilities is maximized, the idea of a critical level leads to critical-level generalized utilitarianism (Broome 2004, Blackorby et al. 2005).

The standard objections to critical-level generalized utilitarianism are the following:

- If we set the critical level at (or below) the individually neutral well-being level, then we get the Repugnant Conclusion (Parfit 1976, 1982, 1984) where, for any population with excellent lives, there is a population with lives barely worth living that is better, provided that the latter includes sufficiently many people,

- If we set the critical level strictly above the individually neutral well-being level, then we get the Very Sadistic Conclusion (Arrhenius 2000, forthcoming) where, for any population with terrible lives not worth living, there is a population with good lives that is worse, provided that the latter includes sufficiently many people.

There are other criteria that avoid the Repugnant and Very Sadistic Conclusions, but they all have their own serious shortcomings. According to average generalized utilitarianism, the contributive value of a life can vary in all respects: a life worth living can have negative contributive value and a life not worth living can have positive contributive value. According to critical-level leximin, as defined by Blackorby et al. (1996), any population with excellent lives is worse than a population with one added individual even when the well-being of all the individuals in the latter population is barely above the critical level. According to critical-level leximin, as suggested by Arrhenius (forthcoming, Section 6.8) and defined in Section 2 of the present paper (Definition 5), any population is worse than a population consisting of one individual, provided that the worst-off individual of the former has lower well-being than the single individual of the latter.

Even in a fixed population framework, where there is no difference between the various generalized utilitarian criteria discussed above and also no difference between the two kinds of leximin criteria, both generalized utilitarianism and leximin have shortcomings if there are many present and future people. If one considers a completely 
egalitarian well-being stream in an intergenerational setting with many future generations and seeks to evaluate whether the present generation should make a sacrifice leading to a uniform benefit of all future generations, then generalized utilitarianism and leximin reach opposite and extreme conclusions: According to generalized utilitarianism, the sacrifice should always be made provided that there are sufficiently many future generations, while according to leximin, it should never be made (cf. Asheim 2010, Section 4.3).

As a response, Zuber and Asheim (2012) have proposed and axiomatized the rankdiscounted generalized utilitarian social welfare order where the weights assigned to (transformed) utility are discounted according to rank, not according to time as in the time-discounted utilitarian criterion. Such rank discounting is solely an expression of inequality aversion and is, therefore, consistent with equal treatment. Still, it allows for a trade-off between the present and the future in the conflict described in the previous paragraph and leads to the same consequences as time-discounted utilitarianism if well-being is perfectly correlated with time. However, if a future generation is expected to be worse off compared to the present, e.g., due to climate change, then that less fortunate future generation will not be assigned less utility weight than the present according to rank-discounted utilitarianism. It will actually receive strictly more utility weight provided there are not infinitely many even worse-off generations. Here we show how the rank-discounted generalized utilitarian approach can fruitfully be extended to the variable population framework. In particular, it avoids shortcomings like the Repugnant and Very Sadistic Conclusions.

In Section 2, we propose the rank-discounted critical level generalized utilitarian (RDCLU) social welfare order (Definition 1). As is the case for Ng's (1989) theory X and Sider's (1991) principle GV, an RDCLU social welfare order is a variable value principle, in the sense that the value of an egalitarian population does not change affinely with population size, and is a context sensitive theory, in the sense that the contributive value of a life depends on the well-being of the rest of the population. However, in contrast to Ng's theory X, the value of each individual depends on its rank, and in contrast to Sider's principle GV, the values are assigned in a prioritarian manner. Then, in Section 3, we provide an axiomatic foundation and show how critical-level generalized utilitarianism (Definition 2) and critical-level leximin (as defined in Definition 5) are the limits of an RDCLU social welfare order for extreme values of the rank utility discount factor.

In Section 4, we present many of the conclusions and principles used to evaluate variable population criteria (cf. Arrhenius forthcoming) and establish how the RDCLU social welfare order avoids the serious objections raised against other variable population criteria. In particular, it escapes both the Repugnant Conclusion and the Very Sadistic Conclusion (while critical-level generalized utilitarianism leads to one of these undesirable conclusions for any critical level), it never assigns positive contributive value to a life not worth living (in contrast to average generalized utilitarianism), and it avoids the Weak Repugnant and Reverse Repugnant Conclusions (which are the conclusions that, in more extreme versions, the two kinds of critical-level leximin lead to). The key intuition is that, with an RDCLU social welfare order, the cumulative effect of adding individuals at a given level of lifetime well-being is bounded. 
Finally, in Section 5, we illustrate in the context of a simple model of optimal population size how the RDCLU social welfare order leads to a smaller population size than critical-level generalized utilitarianism and, in Section 6, note that the problem of accommodating uncertainty still remains. Proofs are contained in the Appendix.

\section{FrameWORK AND DEFINITIONS}

Let $\mathbb{N}$ denote the natural numbers, let $\mathbb{R}$ denote the real numbers, and let, respectively, $\mathbb{R}_{+} / \mathbb{R}_{++} / \mathbb{R}_{-} / \mathbb{R}_{--}$denote the nonnegative/positive/nonpositive/negative real numbers. Let $\mathbf{X}=\cup_{n \in \mathbb{N}} \mathbb{R}^{n}$ be the set of possible finite allocations of lifetime well-being. For every $n \in \mathbb{N}$, each allocation $\mathbf{x} \in \mathbb{R}^{n}$ determines the finite population size, $n(\mathbf{x})=n$, and the distribution of well-being, $\mathbf{x}=\left(x_{1}, \ldots, x_{n(\mathbf{x})}\right)$, among the $n(\mathbf{x})$ individuals that make up the population. This framework suffices, as the binary relations we consider do not depend on the identities of the individuals. Following the usual convention in population ethics, lifetime well-being equal to 0 represents neutrality. Hence, lifetime well-being is normalized so that above neutrality, a life, as a whole, is worth living; below neutrality, it is not.

A social welfare relation (SWR) on the set $\mathbf{X}$ is a binary relation $\succsim$, where for all $\mathbf{x}, \mathbf{y} \in \mathbf{X}, \mathbf{x} \succsim \mathbf{y}$ implies that the allocation $\mathbf{x}$ is deemed socially at least as good as $\mathbf{y}$. Let $\sim$ and $\succ$ denote the symmetric and asymmetric parts of $\succsim$. A complete, reflexive, and transitive SWR is called a social welfare order (SWO).

For each $\mathbf{x} \in \mathbf{X}$, let $\mathbf{x}_{[]}=\left(x_{[1]}, \ldots, x_{[r]}, \ldots, x_{[n(\mathbf{x})]}\right)$ denote the nondecreasing allocation that is a reordering of $\mathbf{x}$; i.e., for each rank $r \in\{1, \ldots, n(\mathbf{x})-1\}, x_{[r]} \leq x_{[r+1]}$. Even though the permutation that turns $\mathbf{x}$ into $\mathbf{x}_{[]}$need not be uniquely determined if different individuals have the same lifetime well-being, the resulting rank-ordered allocation, $\mathbf{x}_{[]}$, is uniquely determined.

For every $n \in \mathbb{N}$ and all $\mathbf{x}, \mathbf{y} \in \mathbb{R}^{n}$, write $\mathbf{x}_{[]} \geq \mathbf{y}_{[]}$whenever $x_{[r]} \geq y_{[r]}$ for all $r \in$ $\{1, \ldots, n(\mathbf{x})\}$, and write $\mathbf{x}_{[]}>\mathbf{y}_{[]}$whenever $\mathbf{x}_{[]} \geq \mathbf{y}_{[]}$and $\mathbf{x}_{[]} \neq \mathbf{y}_{[]}$. Let $(z)_{n} \in \mathbb{R}^{n}$ denote the egalitarian allocation where all $n$ individual have lifetime well-being equal to $z$. Let $(\mathbf{x}, z)$ denote $\mathbf{x} \in \mathbf{X}$ with one added individual with lifetime well-being equal to $z \in \mathbb{R}$. Let $\left(\mathbf{x},(z)_{n}\right)$ denote $\mathbf{x} \in \mathbf{X}$ with $n$ added individuals, all with lifetime well-being equal to $z \in \mathbb{R}$.

We now introduce the SWO proposed, axiomatized, and analyzed in this paper.

DeFINITION 1. An SWR $\succsim$ on $\mathbf{X}$ is a rank-discounted critical-level generalized utilitarian (RDCLU) SWO if there exist $c \in \mathbb{R}_{+}, \beta \in(0,1)$, and a continuous and increasing function $u: \mathbb{R} \rightarrow \mathbb{R}$ such that, for all $\mathbf{x}, \mathbf{y} \in \mathbf{X}$,

$$
\mathbf{x} \succsim \mathbf{y} \Leftrightarrow \sum_{r=1}^{n(\mathbf{x})} \beta^{r}\left(u\left(x_{[r]}\right)-u(c)\right) \geq \sum_{r=1}^{n(\mathbf{y})} \beta^{r}\left(u\left(y_{[r]}\right)-u(c)\right) .
$$

This is a generalized utilitarian criterion, as the function $u$ turns lifetime well-being into transformed values. We refer to the transformed values as utility and refer to $\beta$ as a rank utility discount factor to be consistent with the literature on intertemporal social 
choice. The term "rank-discounted" reflects that the utility weights are not merely rankdependent, but are discounted according to rank by a geometrically decaying function. This is analogous to the use of "time-discounted utilities," rather than "time-dependent utilities," in the time-discounted utilitarian criterion of intertemporal social choice. We refer to $c$ as a critical-level parameter.

We now define established generalized utilitarian SWOs of population ethics.

Definition 2. An SWR $\succsim$ on $\mathbf{X}$ is a critical-level generalized utilitarian (CLU) SWO if there exist $c \in \mathbb{R}_{+}$and a continuous and increasing function $u: \mathbb{R} \rightarrow \mathbb{R}$ such that, for all $\mathbf{x}, \mathbf{y} \in \mathbf{X}$,

$$
\mathbf{x} \succsim \mathbf{y} \quad \Leftrightarrow \quad \sum_{r=1}^{n(\mathbf{x})}\left(u\left(x_{[r]}\right)-u(c)\right) \geq \sum_{r=1}^{n(\mathbf{y})}\left(u\left(y_{[r]}\right)-u(c)\right) .
$$

The CLU SWO has been proposed, discussed, and axiomatized in Blackorby and Donaldson (1984) and Blackorby et al. $(1995,2005)$. The CLU SWO with $c=0$ is referred to as the total generalized utilitarian (TU) SWO.

Definition 3. An SWR $\succsim$ on $\mathbf{X}$ is an average generalized utilitarian (AU) SWO if there exists a continuous and increasing function $u: \mathbb{R} \rightarrow \mathbb{R}$ such that, for all $\mathbf{x}, \mathbf{y} \in \mathbf{X}$,

$$
\mathbf{x} \succsim \mathbf{y} \Leftrightarrow \frac{1}{n(\mathbf{x})} \sum_{r=1}^{n(\mathbf{x})} u\left(x_{[r]}\right) \geq \frac{1}{n(\mathbf{y})} \sum_{r=1}^{n(\mathbf{y})} u\left(y_{[r]}\right) .
$$

The AU SWO is not affected by how $u(0)$ is normalized and thus is not affected by the introduction of a critical-level parameter $c$ either.

Definition 4. An SWR $\succsim$ on $\mathbf{X}$ is a number-dampened critical-level generalized utilitarian (NDCLU) SWO if there exist $c \in \mathbb{R}_{+}$, a function $f: \mathbb{N} \rightarrow \mathbb{R}_{++}$, and a continuous and increasing function $u: \mathbb{R} \rightarrow \mathbb{R}$ such that, for all $\mathbf{x}, \mathbf{y} \in \mathbf{X}$,

$$
\mathbf{x} \succsim \mathbf{y} \Leftrightarrow \frac{f(n(\mathbf{x}))}{n(\mathbf{x})} \sum_{r=1}^{n(\mathbf{x})}\left(u\left(x_{[r]}\right)-u(c)\right) \geq \frac{f(n(\mathbf{y}))}{n(\mathbf{y})} \sum_{r=1}^{n(\mathbf{y})}\left(u\left(y_{[r]}\right)-u(c)\right) .
$$

The NDCLU SWO was introduced in the case with $c=0$ by Ng (1989). It encompasses both the CLU SWO when $f(n)=n$ for all $n \in \mathbb{N}$, and the AU SWO when $f(n)=1$ for all $n \in \mathbb{N}$.

Last, we state a version of a leximin SWO.

Definition 5. An SWR $\succsim$ on $\mathbf{X}$ is a critical-level leximin (CLL) SWO if there exists $c \in \mathbb{R}_{+}$ such that, for all $\mathbf{x}, \mathbf{y} \in \mathbf{X}$ with $n(\mathbf{x}) \geq n(\mathbf{y})$, the following statements hold:

(a) $\mathbf{x} \sim \mathbf{y}$ if and only if $\left(x_{[1]}, \ldots, x_{[n(\mathbf{y})]}\right)=\mathbf{y}_{[]}$and $\left(x_{[n(\mathbf{y})+1]}, \ldots, x_{[n(\mathbf{x})]}\right)=(c)_{n(\mathbf{x})-n(\mathbf{y})}$.

(b) $\mathbf{x} \succ \mathbf{y}$ if and only if (i) there exists $R \in\{1, \ldots, n(\mathbf{y})\}$ such that $x_{[r]}=y_{[r]}$ for all $r \in\{1, \ldots, R-1\}$ and $x_{[R]}>y_{[R]}$ or (ii) $\left(x_{[1]}, \ldots, x_{[n(\mathbf{y})]}\right)=\mathbf{y}_{[]}$and $\left(x_{[n(\mathbf{y})+1]}, \ldots\right.$, $\left.x_{[n(\mathbf{x})]}\right)>(c)_{n(\mathbf{x})-n(\mathbf{y})}$. 
The CLL SWO was suggested by Arrhenius (forthcoming, Section 6.8). It differs, in comparisons of allocations with different population sizes, from the critical-level leximin SWO proposed and axiomatized by Blackorby et al. (1996). ${ }^{1}$

\section{Axioms AND REPRESENTATION RESUltS}

Rank-discounted critical-level generalized utilitarianism can be characterized by the following seven axioms. The first three axioms are sufficient to ensure numerical representation for any fixed population size, and entail that individuals are treated anonymously and with sensitivity for their well-being.

Ахгом 1 (Order). The relation $\succsim$ is complete, reflexive, and transitive on $\mathbf{X}$.

Aхıом 2 (Continuity). For all $n \in \mathbb{N}$ and $\mathbf{x} \in \mathbb{R}^{n}$, the sets $\left\{\mathbf{y} \in \mathbb{R}^{n}: \mathbf{y} \succsim \mathbf{x}\right\}$ and $\left\{\mathbf{y} \in \mathbb{R}^{n}: \mathbf{x} \succsim \mathbf{y}\right\}$ are closed.

Axıом 3 (Suppes-Sen). For all $n \in \mathbb{N}$ and $\mathbf{x}, \mathbf{y} \in \mathbb{R}^{n}$, if $\mathbf{x}_{[]}>\mathbf{y}_{[]}$, then $\mathbf{x} \succ \mathbf{y}$.

While ordinary critical-level generalized utilitarianism allows for unrestricted independence to adding an individual (see Blackorby et al. 2005), our axioms impose such independence only if the added individual is best off (relative to two allocations with the same population size) or worst off.

Axıом 4 (Existence independence of the best off). For all $n \in \mathbb{N}, \mathbf{x}, \mathbf{y} \in \mathbb{R}^{n}$, and $z \in \mathbb{R}$ satisfying $z \geq \max \left\{x_{[n]}, y_{[n]}\right\},(\mathbf{x}, z) \succsim(\mathbf{y}, z)$ if and only if $\mathbf{x} \succsim \mathbf{y}$.

Aхıом 5 (Existence independence of the worst off). For all $\mathbf{x}, \mathbf{y} \in \mathbf{X}$ and $z \in \mathbb{R}$ satisfying $z \leq \min \left\{x_{[1]}, y_{[1]}\right\},(\mathbf{x}, z) \succsim(\mathbf{y}, z)$ if and only if $\mathbf{x} \succsim \mathbf{y}$.

In the spirit of critical-level generalized utilitarianism, we introduce a critical lifetime well-being $c \in \mathbb{R}_{+}$, which if experienced by an added individual without changing the utilities of the existing population, leads to an alternative that is as good as the original. However, the following axiom imposes this if $x_{n(\mathbf{x})} \leq c$, not otherwise. Note that $c$ is at least as large as neutral lifetime well-being.

Aхіом 6 (Existence of a critical level). There exist $c \in \mathbb{R}_{+}$and $n \in \mathbb{N}$ such that, for all $\mathbf{x} \in \mathbb{R}^{n}$ satisfying $x_{[n]} \leq c,(\mathbf{x}, c) \sim \mathbf{x}$.

All axioms above are satisfied also by ordinary critical-level generalized utilitarianism. However, as mentioned in the Introduction and discussed by Arrhenius (forthcoming, Section 5.1), the CLU SWO leads to the Repugnant Conclusion if $c=0$ (adding sufficiently many individuals with lifetime well-being just above 0 makes the

\footnotetext{
${ }^{1}$ Their critical-level leximin SWO is defined as follows: For all $\mathbf{x}, \mathbf{y} \in \mathbf{X}$ with $n(\mathbf{x}) \geq n(\mathbf{y})$ (where $\tilde{\mathbf{y}}$ denotes $\left.\left(\mathbf{y},(c)_{n(\mathbf{x})-n(\mathbf{y})}\right)\right)$, (a) $\mathbf{x} \sim \mathbf{y}$ if and only if $\mathbf{x}_{[]}=\tilde{\mathbf{y}}_{[]}$, and (b) $\mathbf{x} \succ \mathbf{y}$ if and only if there exists $R \in\{1, \ldots, n(\mathbf{x})\}$ such that $x_{[r]}=\tilde{y}_{[r]}$ for all $r \in\{1, \ldots, R-1\}$ and $x_{[R]}>\tilde{y}_{[R]}$.
} 
allocation better than any fixed alternative) and leads to the Very Sadistic Conclusion if $c>0$ (adding sufficiently many individuals with positive lifetime well-being just below $c$ makes the allocation worse than any fixed alternative). Both conclusions rely on the property that adding individuals at a given utility level has unbounded importance. One may consider this property too extreme and rather impose through the following axiom that this need not be the case.

Ахгом 7 (Existence of egalitarian equivalence). For all $\mathbf{x}, \mathbf{y} \in \mathbf{X}$, if $\mathbf{x} \succ \mathbf{y}$, then there exists $z \in \mathbb{R}$ such that, for all $N \in \mathbb{N}, \mathbf{x} \succ(z)_{n} \succ \mathbf{y}$ for some $n \geq N$.

Axiom 7 is the key axiom to avoiding the Repugnant and Very Sadistic Conclusions, while not by itself contradicting these conclusions; thus it is weaker than directly requiring avoidance of the conclusions. For instance, the NDCLU SWO with $f(n)=1$ if $n$ is odd and $f(n)=n$ if $n$ is even satisfies Axiom 7 while leading to the Repugnant Conclusion if $c=0$ and to the Very Sadistic Conclusion if $c>0$.

We now state the result (proven in the Appendix) that these seven axioms characterize the rank-discounted critical-level generalized utilitarian SWO.

THEOREM 1. Consider an $S W R \succsim$ on $\mathbf{X}$. The following two statements are equivalent.

(i) $\succsim$ satisfies Axioms 1-7.

(ii) $\succsim$ is an RDCLU SWO.

It follows from the RDCLU SWO that $c$ is the lifetime well-being that, if experienced by an added individual without changing the utilities of the existing population, leads to an alternative that is as good as the original only if $x_{[n(\mathbf{x})]} \leq c$. If $x_{[n(\mathbf{x})]}>c$, then there is a context-dependent critical level in the open interval $\left(c, x_{[n(\mathbf{x})]}\right)$ that depends on the well-being levels that exceed $c$. This follows from Definition 1, since adding an individual with well-being equal to $x_{[n(\mathbf{x})]}$ increases welfare, while adding an individual with well-being equal to $c$ lowers the weights assigned to individuals at well-being levels that exceed $c$ and thereby reduces welfare.

We end this section by showing how any CLU and CLL SWO is the limit of an RDCLU SWO for extreme values of the rank utility discount factor $\beta$. Write $\succsim_{\beta, u, c}$ for the RDCLU SWO characterized by $\beta, u$, and $c$, write $\succsim_{u, c}$ for the CLU SWO characterized by $u$ and $c$, and write $\succsim_{c}^{L}$ for the CLL SWO characterized by $c$. The following result (proven in the Appendix) establishes that for any nonnegative $c$ and any increasing and continuous function $u$, the CLU SWO $\succsim_{u, c}$ is the limit of the RDCLU SWO $\succsim_{\beta, u, c}$ as $\beta$ approaches 1 , and the CLL SWO $\succsim_{c}^{L}$ is the limit of the RDCLU SWO $\succsim_{\beta, u, c}$ as $\beta$ approaches 0 . Note that in the case of $\succsim_{c}^{L}$, the weak preference of $\succsim_{\beta, u, c}$ for small $\beta$ is both sufficient and necessary.

THeOREM 2. For any $c \in \mathbb{R}_{+}$, and any continuous and increasing function $u$, the following results hold for any $\mathbf{x}, \mathbf{y} \in \mathbf{X}$ : 
(i) $\mathbf{x} \succsim_{u, c} \mathbf{y}$ if there exists $\bar{\beta} \in(0,1)$ such that, for all $\beta \in(\bar{\beta}, 1), \mathbf{x} \succsim_{\beta, u, c} \mathbf{y}$.

(ii) $\mathbf{x} \succsim_{c}^{L} \mathbf{y}$ if and only if there exists $\underline{\beta} \in(0,1)$ such that for all $\beta \in(0, \underline{\beta}), \mathbf{x} \succsim_{\beta, u, c} \mathbf{y}$.

The case where the rank-discount factor $\beta$ approaches 0 , and thereby an RDCLU SWO approaches a CLL SWO, is related to the case where a given allocation is replicated in the following sense: For any $\mathbf{x} \in \mathbf{X}$ and any $k \in \mathbb{N}$, the $k$-replica of $\mathbf{x}$ is an allocation $\mathbf{x}^{k}$ with $k n(\mathbf{x})$ individuals having the property that $x_{[r]}=x_{[\rho]}^{k}$ for all $r \in\{1, \ldots, n(\mathbf{x})\}$ and $\rho \in\{k(r-1)+1, \ldots, k r\}$. Theorem 2 implies that for fixed $\mathbf{x}, \mathbf{y} \in \mathbf{X}$ and $\beta \in(0,1)$, there exists $K \in \mathbb{N}$ such that for all $k>K, \mathbf{x}^{k} \succsim_{\beta, u, c} \mathbf{y}^{k}$ if and only if $\mathbf{x} \succsim_{c}^{L} \mathbf{y}$. Hence, as a given allocation is replicated, utility weight is redistributed toward the individuals with lowest lifetime well-being.

\section{Evaluating RDCLU}

We now compare the RDCLU SWO to other criteria by means of conditions used in the literature on population ethics. In particular, we establish two results that show how the RDCLU SWO compares favorably to the NDCLU SWO for any function $f$ (cf. Definition 4). The conditions are referred to by the labels used by Arrhenius (forthcoming), but are formulated here in a precise manner using the terminology of this paper.

According to Parfit $(1976,1982,1984)$, an SWR leads to the Repugnant Conclusion if for any egalitarian allocation with very high positive well-being, there is an egalitarian allocation with very low positive well-being that is better.

An SWR $\succsim$ leads to the Repugnant Conclusion if, for all $y, z \in \mathbb{R}$ with $y>z>0$ and $k \in \mathbb{N}$, there is $n>k$ such that $(y)_{k} \prec(z)_{n}{ }^{2}$

The TU SWO has been criticized for not avoiding this conclusion.

Besides, if $c>0$, the CLU SWO is subject to the following weaker form of the Repugnant Conclusion, namely that for any egalitarian allocation with very high positive well-being, there is an egalitarian allocation with well-being just above the critical level that is better:

An SWR $\succsim$ leads to the Weak Repugnant Conclusion if, for all $y, z \in \mathbb{R}$ with $y>z>c$ and $k \in \mathbb{N}$, there is $n>k$ such that $(y)_{k} \prec(z)_{n}$.

As pointed out by Arrhenius (2000, forthcoming), the CLU SWO with $c>0$ also leads to the Very Sadistic Conclusion that, for any allocation with negative well-being, there is an egalitarian allocation with positive well-being that is worse.

\footnotetext{
${ }^{2}$ This definition is equivalent to the one provided by Blackorby et al. (2005, p. 162). If one determines disjoint nonempty intervals $\left(0, x^{\ell}\right]$ and $\left[x^{h}, \infty\right)$ with, respectively, very low and very high positive wellbeing, then the Repugnant Conclusion might alternatively be defined as the property that, for all $y \in\left[x^{h}, \infty\right)$ and $k \in \mathbb{N}$, there are $z \in\left(0, x^{\ell}\right]$ and $n>k$ such that $(y)_{k} \prec(z)_{n}$. With this alternative definition, the CLU SWO avoids the Repugnant Conclusion if $c \geq x^{\ell}$, but not if $c \in\left(0, x^{\ell}\right)$, while the RDCLU SWO still avoids the Repugnant Conclusion for all $c \geq 0$ (just pick $k$ large enough to ensure $\left(1-\beta^{k}\right) u\left(x^{h}\right) \geq u\left(x^{\ell}\right)$ ).
} 
An SWR $\succsim$ leads to the Very Sadistic Conclusion if, for all $\mathbf{x} \in \mathbf{X}$, there are $z \in \mathbb{R}_{++}$and $n \in \mathbb{N}$ such that $\mathbf{x} \succ(z)_{n}$.

This follows since if $0<z<c$, then by choosing $n$ sufficiently large, $(z)_{n}$ can be made worse than any given allocation $\mathbf{x}$, even when the individuals in $\mathbf{x}$ have very negative lifetime well-being.

As an alternative to avoiding the Very Sadistic Conclusion, Arrhenius (forthcoming) has also proposed the Weak Non-Sadism Condition:

An SWR $\succsim$ satisfies the Weak Non-Sadism Condition if there are $y \in \mathbb{R}_{--}$and $k \in \mathbb{N}$ such that for all $\mathbf{x} \in \mathbf{X}, z \in \mathbb{R}_{++}$and $n \in \mathbb{N},\left(\mathbf{x},(y)_{k}\right) \precsim\left(\mathbf{x},(z)_{n}\right)$.

The RDCLU avoids the Weak Repugnant Conclusion, even when $c=0$, implying that it also escapes the Repugnant Conclusion for all $c \geq 0$. It also avoids the Very Sadistic Conclusion and, provided that the function $u$ is bounded above, satisfies the Weak NonSadism Condition. This is in contrast to the large class of generalized utilitarian criteria embodied in the NDCLU SWO: these criteria necessarily violate the Weak Non-Sadism Condition or imply the Weak Repugnant Conclusion, as stated in the following result (proven in the Appendix).

Proposition 1. (i) An NDCLU SWO either leads to the Weak Repugnant Conclusion or violates the Weak Non-Sadism Condition, even when the function $u$ is bounded above.

(ii) An RDCLU SWO avoids the Weak Repugnant Conclusion and satisfies the Weak Non-Sadism Condition, provided the function u is bounded above.

Being able to avoid the Weak Repugnant Conclusion while satisfying the Weak NonSadism Condition, the RDCLU SWO is rather remarkable. The NDCLU SWO is indeed one of the most general forms that have been proposed. ${ }^{3}$

Outside the class of generalized utilitarian criteria, Blackorby et al. (1996) have proposed a critical-level leximin SWO (cf. footnote 1). It, however, leads to the following more extreme version of the Weak Repugnant Conclusion: For all $y, z \in \mathbb{R}$ with $y>z>c$ and $k, n \in \mathbb{N}$ with $k<n,(y)_{k} \prec(z)_{n}$. Thus it does not escape the impossibility encountered by the NDCLU SWO.

A version of Proposition 1 that restricts attention to the more undesirable implications of variable population criteria-the Repugnant and Very Sadistic Conclusions-is obtained if one adopts the following natural requirement (called Negative Expansion Principle by Blackorby et al. 2005):

\footnotetext{
${ }^{3} \mathrm{~A}$ restricted number-dampened generalized utilitarian (RNDU) SWO is attributed to Thomas Hurka by Blackorby et al. (2005). It is similar to the NDCLU SWO with $c=0$, except that $f(n)=n$ if average well-being is negative. Considering the RNDU SWO would not alter our result, because the proof of Proposition 1 only involves streams with positive average well-being. Another family is number-sensitive critical-level generalized utilitarianism (Blackorby et al. 2002, 2005). It is similar to the CLU except that the critical level changes with population size. It leads to the Very Sadistic Conclusion and a modified version of the Weak Repugnant Conclusion.
} 
An SWR $\succsim$ satisfies the Negative Mere Addition Principle if, for all $\mathbf{x} \in \mathbf{X}$ and $z \in \mathbb{R}_{--}$, $(\mathbf{x}, z) \prec \mathbf{x}$.

This property is satisfied by the RDCLU SWO, but not by some utilitarian criteria like the AU SWO. And we have the following result (proven in the Appendix).

Proposition 2. (i) An NDCLU SWO satisfying the Negative Mere Addition Principle leads either to the Repugnant Conclusion or to the Very Sadistic Conclusion.

(ii) An RDCLU SWO avoids the Repugnant Conclusion and the Very Sadistic Conclusion, and satisfies the Negative Mere Addition Principle.

Contrary to Proposition 1, Proposition 2 does not depend on the function $u$ being bounded above for the RDCLU SWO to satisfy all desirable properties.

Besides the RDCLU SWO, the CLL SWO also avoids all the above shortcomings. However, the CLL SWO leads to the problematic conclusion that for any egalitarian allocation with very high positive well-being, there is a better one-individual allocation with slightly higher well-being:

An SWR $\succsim$ leads to the Reverse Repugnant Conclusion if, for all $y \in \mathbb{R}_{++}$and $k \in \mathbb{N}$, $(y)_{k} \prec(z)_{1}$ if $y<z$.

This conclusion does not follow from the RDCLU SWO as

$$
\frac{1-\beta^{k}}{1-\beta}(u(y)-u(c)) \geq(u(z)-u(c)),
$$

and thus $(y)_{k} \succsim(z)_{1}$, according to the RDCLU SWO, is clearly consistent with $c<y<z$, provided that $z$ is sufficiently close to $y$ and $k$ is sufficiently large. The conclusion does not follow from the CLU SWO either, but it follows from the AU SWO. The CLL SWO leads even to the following more extreme version of the Reverse Repugnant Conclusion: For all $\mathbf{x} \in \mathbf{X}, \mathbf{x} \prec(z)_{1}$ if $x_{[1]}<z$. We believe that this disqualifies the CLL SWO as a satisfying criterion.

Propositions 1 and 2 build a strong case in favor of the RDCLU SWO. It is, however, interesting to further assess the criterion with respect to other principles proposed in the literature on variable population criteria.

As shown in Arrhenius (forthcoming, Section 3.8), both TU and AU SWOs fail the following principle:

An SWR $\succsim$ satisfies the Strong Quality Addition Principle if there are $y, z \in \mathbb{R}$ with $y>z>0$

and $k \in \mathbb{N}$ such that for all $\mathbf{x} \in \mathbf{X}$ and $n>k,\left(\mathbf{x},(y)_{k}\right) \succsim\left(\mathbf{x},(z)_{n}\right)$.

In contrast, this principle is satisfied by the CLL SWO and also by the CLU SWO with $c>0$ (just let $y>c>z>0$, implying that $\left(\mathbf{x},(y)_{k}\right) \succ\left(\mathbf{x},(z)_{n}\right)$ for all $\mathbf{x} \in \mathbf{X}$ and $k, n \in \mathbb{N}$ according to the CLU SWO).

To show that the Strong Quality Addition Principle is satisfied by the RDCLU SWO with $c \geq 0$ (i.e., even if $c=0$ ) is more delicate: Let $y, z \in \mathbb{R}$ and $k \in \mathbb{N}$ satisfy the requirements that $y>z>c$ and

$$
\frac{1-\beta^{k}}{1-\beta}(u(y)-u(c)) \geq \frac{1}{1-\beta}(u(z)-u(c)) .
$$


Then clearly $\left(\mathbf{x},(y)_{k}\right) \succ\left(\mathbf{x},(z)_{n}\right)$ according to the RDCLU SWO for all $n \in \mathbb{N}$ if $x_{[n(\mathbf{x})]} \leq z$. Moreover, with $n>k$, raising elements of $\mathbf{x}$ above $z$ contributes more to $\left(\mathbf{x},(y)_{k}\right)$ than to $\left(\mathbf{x},(z)_{n}\right)$ as their ranks are lower in the former allocation than in the latter, implying that the strict preference cannot be reversed.

Arrhenius (forthcoming) suggests the following weak version of the Pigou-Dalton transfer principle, which is satisfied if any given allocation is deemed worse than an egalitarian allocation with the same number of individuals and higher average (and thus higher total) well-being.

An SWR $\succsim$ satisfies the Non-Anti-Egalitarianism Principle if, for all $\mathbf{x} \in \mathbf{X}$ and $z>$ $(1 / n(\mathbf{x})) \sum_{r=1}^{n(\mathbf{x})} x_{[r]}, \mathbf{x} \prec(z)_{n(\mathbf{x})}$.

This principle is satisfied by the RDCLU SWO if $u$ satisfies $\beta \times \mathcal{C}_{u} \leq 1$, where

$$
\mathcal{C}_{u}=\sup _{0<\varepsilon \leq x \leq x^{\prime}} \frac{u\left(x^{\prime}+\varepsilon\right)-u\left(x^{\prime}\right)}{u(x)-u(x-\varepsilon)}
$$

is an index of nonconcavity of the function $u .{ }^{4}$ It is also satisfied by CLU and AU SWOs if $u$ is concave and, of course, by the CLL SWO.

Last, the RDCLU, CLU (if $c>0$ ), AU, and CLL SWOs all violate the following principle:

An SWR $\succsim$ satisfies the Mere Addition Principle if, for all $\mathbf{x} \in \mathbf{X}$ and $z \in \mathbb{R}_{++},(\mathbf{x}, z) \succsim \mathbf{x}$.

Of the SWRs we consider, only the NDCLU SWO with $c=0$ and for a restricted set of the function $f$, including the TU SWO, satisfies the Mere Addition Principle. The RDCLU SWO with $c=0$ does not, because adding an individual with low positive well-being will decrease the weights on individuals with higher well-being and might thereby worsen the allocation. ${ }^{5}$

With the possible exception of the Mere Addition Principle, this shows that the RDCLU SWO has desirable properties when evaluated by the above conditions and principles. These positive conclusions are of interest in view of Arrhenius (forthcoming, Section 11.14), where it is shown that the Weak Non-Sadism Condition is hard to satisfy unless other desirable properties are given up.

Table 1 summarizes the different principles and conclusions satisfied by the different SWOs discussed in this section, where the plus sign (+) denotes that the principle is satisfied (or the conclusion follows or, in the first three lines and the sixth, the conclusion is avoided) and the minus sign (-) denotes the opposite.

\section{Optimal population Size}

Following Dasgupta (1988, pp. 123-125), let $m$ be the total available amount of a consumption good and, as before, let $n$ denote the number of individuals. Let the well-being

\footnotetext{
${ }^{4}$ See the proof of Proposition 6 in Zuber and Asheim (2012).

${ }^{5}$ Indeed, adding people with positive but very low well-being may increase inequality, an objection that is often made against the Mere Addition Principle. See, for instance, Carlson (1998), who also proves that the Mere Addition Principle and the Non-Anti-Egalitarianism Principle imply a conclusion similar to the Repugnant Conclusion.
} 


\begin{tabular}{|c|c|c|c|c|c|}
\hline & $\begin{array}{c}\text { RDCLU } \\
c \geq 0\end{array}$ & $\begin{array}{l}\text { CLU } \\
c=0\end{array}$ & $\begin{array}{l}\text { CLU } \\
c>0\end{array}$ & $\mathrm{AU}$ & $\begin{array}{l}\text { CLL } \\
c \geq 0\end{array}$ \\
\hline \multicolumn{6}{|l|}{ Avoiding the } \\
\hline Repugnant Conclusion & + & - & + & + & + \\
\hline \multicolumn{6}{|l|}{ Avoiding the Weak } \\
\hline Repugnant Conclusion & + & - & - & + & + \\
\hline \multicolumn{6}{|l|}{ Avoiding the Very } \\
\hline Sadistic Conclusion & + & + & - & + & + \\
\hline \multicolumn{6}{|l|}{ Weak Non-Sadism } \\
\hline Condition & $+^{\mathrm{a}}$ & + & - & - & + \\
\hline \multicolumn{6}{|l|}{ Negative Mere Addition } \\
\hline Principle & + & + & + & - & + \\
\hline \multicolumn{6}{|l|}{ Avoiding the Reverse } \\
\hline Repugnant Conclusion & + & + & + & - & - \\
\hline \multicolumn{6}{|l|}{ Strong Quality Addition } \\
\hline Principle & + & - & + & - & + \\
\hline \multicolumn{6}{|l|}{ Non-Anti-Egalitarianism } \\
\hline Principle & $+{ }^{b}$ & $+^{\mathrm{c}}$ & $+{ }^{c}$ & $+^{\mathrm{c}}$ & + \\
\hline \multicolumn{6}{|l|}{ Mere Addition } \\
\hline Principle & - & + & - & - & - \\
\hline
\end{tabular}

aff $\{u(z): z \in \mathbb{R}\}$ is bounded above.

${ }^{\mathrm{b}}$ If $\beta \times \mathcal{C}_{u} \leq 1$.

${ }^{\mathrm{c}}$ If $u$ is concave.

TABLE 1. Population principles and social welfare orders.

of each individual be equal to allocated consumption minus $s$, implying that $s$ is the level of consumption needed to attain neutrality. Hence, a life is worth living if consumption exceeds $s$, while it is not if consumption falls below $s$.

Under the AU SWO, the optimal population size $n$ is equal to 1 , as this maximizes average utility. Likewise for the CLL SWO, as this maximizes the utility of the worst-off individual. Turn now to the CLU and RDCLU SWOs. Under the assumption that $u$ is concave, it is optimal to divide the available amount $m$ equally among the $n$ individuals. Hence, the so-called genesis problem is to optimize $n$ given that each individual's well-being $x(n)$ equals $(m / n)-s$, with $n$ treated as a continuous variable, for tractability. Dasgupta (2005) argues that the genesis problem might not be the most interesting problem for population ethics. It is also different from the problem studied by Palivos and Yip (1993) and Razin and Yuen (1995), where the development of per capita wellbeing and population size is optimized within models of economic growth. Still, it is illustrative and leads to generalizable insights (cf. Dasgupta 1988, footnote 16).

Under the CLU SWO, the genesis problem becomes

$$
\max _{n} n\left(u\left(\frac{m}{n}-s\right)-u(c)\right)
$$

leading to the first-order condition

$$
u(x(n))-u(c)=(x(n)+s) u^{\prime}(x(n)) .
$$


If $u(x)=(1 /(1-\eta))(x+s)^{1-\eta}$ with $\eta>1$, then (1) can be transformed to

$$
\frac{x(n)+s}{c+s}=\eta^{1 /(\eta-1)} .
$$

As the elasticity of marginal utility $\eta$ goes to infinity, $\eta^{1 /(\eta-1)}$ goes to unity, illustrating how the CLU SWO leads to the Repugnant Conclusion if $c=0$ and to the Weak Repugnant Conclusion otherwise.

Under the RDCLU SWO, the genesis problem becomes

$$
\max _{n} \frac{1-\beta^{n}}{1-\beta}\left(u\left(\frac{m}{n}-s\right)-u(c)\right),
$$

leading to the first-order condition

$$
\gamma(n, \beta)(u(x(n))-u(c))=(x(n)+s) u^{\prime}(x(n)),
$$

where

$$
\gamma(n, \beta):=\frac{\beta^{n}\left(-\ln \beta^{n}\right)}{1-\beta^{n}}
$$

can be shown to satisfy $0<\gamma(n, \beta)<1, \partial \gamma / \partial n<0, \partial \gamma / \partial \beta>0$, and $\lim _{\beta \rightarrow 1} \gamma(n, \beta)=1$. If $u(x)=(1 /(1-\eta))(x+s)^{1-\eta}$ with $\eta>1$, then (2) can be transformed to

$$
\frac{x(n)+s}{c+s}=\left(\frac{\gamma(n, \beta)+\eta-1}{\gamma(n, \beta)}\right)^{1 /(\eta-1)} .
$$

The left-hand side (1.h.s.) is a decreasing function of $n$ that equals 1 for $n=m /(c+s)$ and approaches $\infty$ as $n \downarrow 0$. The right-hand side (r.h.s.) is greater than $\eta^{1 /(\eta-1)}>1$ and an increasing function of $n$-implying that the first-order condition determines a unique optimal value of $n$-and a decreasing function of $\beta$ that approaches $\eta^{1 /(\eta-1)}$ as $\beta \rightarrow 1-$ implying that this optimal population size is lower under the RDCLU SWO than under the CLU SWO. Thus, this analysis illustrates how the RDCLU SWO leads to an escape from the Repugnant Conclusion.

\section{Concluding Remarks}

We have contributed to population ethics by proposing and axiomatizing the rankdiscounted critical-level generalized utilitarian (RDCLU) SWO. By doing so we have taken one step toward preparing the rank-discounted utilitarian criterion (see Zuber and Asheim 2012) for practical use.

First of all, we have generalized rank-discounted utilitarianism by letting individuals rather than generations be the object of analysis. This generalization has several implications, one of which is particularly interesting to point out: If there is no intragenerational inequality and per capita well-being increases over time, then the aggregate marginal utility of a generation increases with the number of individuals belonging to this generation. On the other hand, the average rank-dependent discount rate with 
which this aggregate marginal utility is discounted between this generation and its immediate predecessor increases with its size.

Second, we have allowed for analysis of a situation where population changes endogenously, e.g., as a consequence of climate change. By introducing a critical level that if experienced by an added individual without changing the utilities of the existing population leads to an alternative that is as good as the original only if the well-being levels of the existing population do not exceed the critical level, we have been able to combine critical-level population ethics with rank-discounted utilitarianism in an appealing manner. In particular, the RDCLU SWO offers an escape from the Repugnant and Very Sadistic Conclusions.

However, practical application of the rank-discounted utilitarian criterion also requires explicit treatment of risk. This is the topic of Asheim and Zuber (2014).

\section{APpendix: Proofs}

We first prove the representation result by showing that statements (i) and (ii) of Theorem 1 are equivalent. It is straightforward to show that an RDCLU SWO satisfies Axioms $1-7$, so that statement (ii) implies statement (i). Hence, to prove Theorem 1, we need to show that statement (i) implies statement (ii); that is, that an SWR $\succsim$ on $\mathbf{X}$ satisfying Axioms 1-7 is an RDCLU SWO. This is shown by means of Lemmas 1-4.

We define the restriction $\succsim_{n}$ of $\succsim$ to $\mathbb{R}^{n}$ in the following way: for all $\mathbf{x}, \mathbf{y} \in \mathbb{R}^{n}, \mathbf{x} \succsim_{n} \mathbf{y}$ if and only if $\mathbf{x} \succsim \mathbf{y}$. We begin by establishing a representation result for sets of allocations with the same finite population size.

Lemma 1. If the SWR $\succsim$ satisfies Axioms 1-5, then there exist $\beta \in \mathbb{R}_{++}$and a continuous increasing function $u: \mathbb{R} \rightarrow \mathbb{R}$ such that for all $n \in \mathbb{N}$ and $\mathbf{x}, \mathbf{y} \in \mathbb{R}^{n}$,

$$
\mathbf{x} \succsim_{n} \mathbf{y} \quad \Leftrightarrow \quad \sum_{r=1}^{n} \beta^{r} u\left(x_{[r]}\right) \geq \sum_{r=1}^{n} \beta^{r} u\left(y_{[r]}\right) .
$$

Proof. For any $n \geq 3$, we show that the relation $\succsim_{n}$ satisfies the following properties proposed by Ebert (1988): continuous order, monotonicity, symmetry, and independence with respect to ordered vectors. By Ebert (1988, Theorem 1), this implies that there exist continuous increasing functions $u_{r}^{n}$ such that for all $n \in \mathbb{N}$ and $\mathbf{x}, \mathbf{y} \in \mathbb{R}^{n}$,

$$
\mathbf{x} \succsim_{n} \mathbf{y} \quad \Leftrightarrow \quad \sum_{r=1}^{n} u_{r}^{n}\left(x_{[r]}\right) \geq \sum_{r=1}^{n} u_{r}^{n}\left(y_{[r]}\right) .
$$

The fact that $\succsim_{n}$ is a continuous order follows from Axioms 1 and 2 . The fact that it satisfies monotonicity and symmetry follows from Axiom 3 . For the independence condition, we can apply Gorman's (1968) theorem on the ordered set $\left\{\mathbf{x} \in \mathbb{R}^{n}: x(1) \leq\right.$ $\cdots \leq x(n)\}$. By Axiom 4 , we know that all sets $\{1,2, \ldots, t\}$ for $1<t<n$ are separable. By Axiom 5, we know that all sets $\{t, t+1, \ldots, n\}$ for $1<t<n$ are separable. By intersections of such separable subsets, we can obtain any subsets $\{t, t+1\}, 1 \leq t<n$, which are 
therefore separable by Gorman's (1968) theorem. By unions of such subsets, we can obtain any subset of indices in $\{1, \ldots, n\}$ so that they are also separable by Gorman's (1968) theorem. This corresponds to Ebert's (1988) Independence with Respect to Ordered Vectors. We may set $u_{r}^{n}(0)=0$ for all $r \leq n$; this normalization can be performed without loss of generality because it leaves the ranking unchanged.

Now, representation (A.2) exists for $\succsim_{n}$ whatever $n \in \mathbb{N}$. Furthermore, by Axiom 4 , we have the following equivalences (for $z \geq\left\{x_{[n]}, y_{[n]}\right\}$ ):

$$
\begin{aligned}
\sum_{r=1}^{n} u_{r}^{n}\left(x_{[r]}\right) \geq \sum_{r=1}^{n} u_{r}^{n}\left(y_{[r]}\right) & \Leftrightarrow \quad \mathbf{x} \succsim_{n} \mathbf{y} \\
& \Leftrightarrow \quad(\mathbf{x}, z) \succsim_{n+1}(\mathbf{y}, z) \\
& \Leftrightarrow \sum_{r=1}^{n} u_{r}^{n+1}\left(x_{[r]}\right)+u_{n+1}^{n+1}(z) \geq \sum_{r=1}^{n} u_{r}^{n+1}\left(y_{[r]}\right)+u_{n+1}^{n+1}(z) .
\end{aligned}
$$

By standard uniqueness results for additive functions on rank-ordered sets, we can take (after the appropriate normalization) $u_{r}^{n} \equiv u_{r}^{n+1}$. We can henceforth drop the superscript $n$ in functions $u_{r}^{n}$.

By Axiom 5, we have the following equivalences (for $z \leq \min \left\{x_{[n]}, y_{[n]}\right\}$ ):

$$
\begin{aligned}
\sum_{r=1}^{n} u_{r}\left(x_{[r]}\right) \geq \sum_{r=1}^{n} u_{r}\left(y_{[r]}\right) & \Leftrightarrow \quad \mathbf{x} \succsim_{n} \mathbf{y} \\
& \Leftrightarrow \quad(\mathbf{x}, z) \succsim_{n+1}(\mathbf{y}, z) \\
& \Leftrightarrow \sum_{r=1}^{n} u_{r+1}\left(x_{[r]}\right)+u_{1}(z) \geq \sum_{r=1}^{n} u_{r+1}\left(y_{[r]}\right)+u_{1}(z) .
\end{aligned}
$$

By the cardinality of the additive representation and the normalization condition, there must exist a $\beta>0$ such that $u_{r+1}(y)=\beta u_{r}(y)$ for any $y \in \mathbb{R}$. Note that $\beta$ does not depend on $r$. We obtain (A.1), where $u \equiv u_{1}$ is a continuous increasing function from $\mathbb{R}$ to $\mathbb{R}$.

By Axiom 4, the proof can be extended to the case where $n=1$ or $n=2$.

Let $c \in \mathbb{R}_{+}$be the critical-level parameter introduced in Axiom 6. Define $\mathbf{X}_{c}$ as the nonempty set $\left\{\mathbf{x} \in \mathbf{X}: \mathbf{x}_{[]} \leq(c)_{n(\mathbf{x})}\right\}$ of finite allocations where well-being does not exceed $c$. We obtain a representation result for finite allocations (with variable population) where well-being does not exceed $c$.

Lemma 2. If the SWR $\succsim$ satisfies Axioms 1-6 and $c \in \mathbb{R}_{+}$is the critical-level parameter of Axiom 6 , then there exist $\beta \in \mathbb{R}_{++}$and a continuous increasing function $u: \mathbb{R} \rightarrow \mathbb{R}$ such that, for all $\mathbf{x}, \mathbf{y} \in \mathbf{X}_{c}$,

$$
\mathbf{x} \succsim \mathbf{y} \Leftrightarrow \sum_{r=1}^{n(\mathbf{x})} \beta^{r}\left(u\left(x_{[r]}\right)-u(c)\right) \geq \sum_{r=1}^{n(\mathbf{y})} \beta^{r}\left(u\left(y_{[r]}\right)-u(c)\right) .
$$


Proof. Assume that $\mathbf{x}, \mathbf{y} \in \mathbf{X}_{c}$ and $n(\mathbf{x}) \leq n(\mathbf{y})$, and let $k=n(\mathbf{y})-n(\mathbf{x})$. Then

$$
\begin{aligned}
\mathbf{x} \succsim \mathbf{y} & \Leftrightarrow \quad\left(\mathbf{x},(c)_{k}\right){ }_{n}(\mathbf{y}) \mathbf{y} \\
& \Leftrightarrow \sum_{r=1}^{n(\mathbf{x})} \beta^{r} u\left(x_{[r]}\right)+\sum_{r=n(\mathbf{x})+1}^{n(\mathbf{y})} \beta^{r} u(c) \geq \sum_{r=1}^{n(\mathbf{y})} \beta^{r} u\left(y_{[r]}\right) \\
& \Leftrightarrow \sum_{r=1}^{n(\mathbf{x})} \beta^{r} u\left(x_{[r]}\right)+\sum_{r=n(\mathbf{x})+1}^{n(\mathbf{y})} \beta^{r} u(c)-\sum_{r=1}^{n(\mathbf{y})} \beta^{r} u(c) \geq \sum_{r=1}^{n(\mathbf{y})} \beta^{r} u\left(y_{[r]}\right)-\sum_{r=1}^{n(\mathbf{y})} \beta^{r} u(c) \\
& \Leftrightarrow \sum_{r=1}^{n(\mathbf{x})} \beta^{r}\left(u\left(x_{[r]}\right)-u(c)\right) \geq \sum_{r=1}^{n(\mathbf{y})} \beta^{r}\left(u\left(y_{[r]}\right)-u(c)\right)
\end{aligned}
$$

since $\mathbf{x} \sim\left(\mathbf{x},(c)_{k}\right)$ by $k$ applications of Axiom 6, using Axiom 5 repeatedly to ensure that the allocation is in $\mathbb{R}^{n}$ when Axiom 6 is applied.

The following lemma shows that adding Axiom 7 implies that the rank utility discount factor, $\beta$, is smaller than 1 .

Lемма 3. If the SWR $\succsim$ satisfies Axioms $1-7$ and $c \in \mathbb{R}_{+}$is the critical-level parameter of Axiom 6, then there exist $0<\beta<1$ and a continuous increasing function $u: \mathbb{R} \rightarrow \mathbb{R}$ such that for all $\mathbf{x}, \mathbf{y} \in \mathbf{X}_{c}$,

$$
\mathbf{x} \succsim \mathbf{y} \Leftrightarrow \sum_{r=1}^{n(\mathbf{x})} \beta^{r}\left(u\left(x_{[r]}\right)-u(c)\right) \geq \sum_{r=1}^{n(\mathbf{y})} \beta^{r}\left(u\left(y_{[r]}\right)-u(c)\right) .
$$

Proof. Let $x, y \in \mathbb{R}$ satisfy that $c \geq x>y$ and let $k \in \mathbb{N}$. Assume that there exist $z \in \mathbb{R}$ and $\ell \in \mathbb{N}$ such that $(x)_{k} \succ(z)_{\ell} \succ(y)_{k}$ (note that $z<c$, because otherwise, by Axioms 1 , 3 , and $6,(z)_{\ell} \succsim(c)_{\ell} \sim(c)_{k} \succsim(x)_{k}$, a contradiction). By Lemma 2, this means that (for $\beta \neq 1$; the case $\beta=1$ can be treated similarly)

$$
\frac{\beta^{k}-1}{\beta-1}(u(x)-u(c))>\frac{\beta^{\ell}-1}{\beta-1}(u(z)-u(c))>\frac{\beta^{k}-1}{\beta-1}(u(y)-u(c)) .
$$

When $\beta>1, \lim _{\ell \rightarrow \infty}\left(\beta^{\ell}-1\right) /(\beta-1)=\infty$ so that

$$
\lim _{\ell \rightarrow \infty} \frac{\beta^{\ell}-1}{\beta-1}(u(z)-u(c))=-\infty .
$$

Hence there exists $N>\ell$ such that

$$
\frac{\beta^{n}-1}{\beta-1}(u(z)-u(c))<\frac{\beta^{n(\mathbf{x})}-1}{\beta-1}(u(y)-u(c))
$$

for all $n \geq N$, a contradiction of Axiom 7 . 
Finally, we extend the representation to the entire domain $\mathbf{X}$ of all finite allocations by showing that any finite allocation $\mathbf{x}$ can be made as bad as an allocation where all individuals are at the critical level $c$ by adding sufficiently many people at a low wellbeing level $z$, and thus indifferent to an egalitarian allocation where each individual's well-being equals $x \leq c$. This allows us to combine the representations of Lemmas 1 and 2, and shows that statement (i) of Theorem 1 implies statement (ii).

Lemma 4. If the SWR $\succsim$ satisfies Axioms $1-7$, then there exist $0<\beta<1$ and a continuous increasing function $u: \mathbb{R} \rightarrow \mathbb{R}$ such that for all $\mathbf{x}, \mathbf{y} \in \mathbf{X}$,

$$
\mathbf{x} \succsim \mathbf{y} \quad \Leftrightarrow \quad \sum_{r=1}^{n(\mathbf{x})} \beta^{r}\left(u\left(x_{[r]}\right)-u(c)\right) \geq \sum_{r=1}^{n(\mathbf{y})} \beta^{r}\left(u\left(y_{[r]}\right)-u(c)\right) .
$$

Proof. Step 1: For any $n \in \mathbb{N}, \mathbf{x} \in \mathbb{R}^{n}$, and $z \in \mathbb{R}$ satisfying $z \leq x_{[1]}$ and $z<c$, there exists $k(\mathbf{x})$ such that for all $k \geq k(\mathbf{x}),\left(\mathbf{x},(z)_{k}\right) \prec(c)_{n+k}$.

By Lemma 3, we know that $\beta<1$ in the representation on $\mathbf{X}_{c}$. By Lemma 1, the property extends to $\mathbb{R}^{n}$ for any $n \in \mathbb{N}$.

For any $\mathbf{x} \in \mathbb{R}^{n}$, the $n$-equally distributed equivalent of $\mathbf{x}$, denoted $e_{n}(\mathbf{x})$, is the real number $x \in \mathbb{R}$ such that $(x)_{n} \sim_{n} \mathbf{x}$. Axioms 1-3 imply that $e_{n}: \mathbb{R}^{n} \rightarrow \mathbb{R}$ is well defined. By Lemma 1 and since Axioms 1-5 hold, it is defined as

$$
e_{n}(\mathbf{x})=u^{-1}\left(\frac{1-\beta}{1-\beta^{n}} \sum_{r=1}^{n} \beta^{r-1} u\left(x_{[r]}\right)\right) .
$$

Now let $z \leq x_{[1]}$ and $z<c$. We obtain the expression

$$
\begin{aligned}
e_{n+k}\left(\mathbf{x},(z)_{k}\right) & =u^{-1}\left(\frac{1-\beta}{1-\beta^{n+k}}\left(\sum_{r=1}^{k} \beta^{r-1} u(z)+\sum_{r=k+1}^{n+k} \beta^{r-1} u\left(x_{[r]}\right)\right)\right) \\
& =u^{-1}\left(\frac{1-\beta^{k}}{1-\beta^{n+k}} u(z)+\frac{\beta^{k}-\beta^{n+k}}{1-\beta^{n+k}} \cdot \frac{1-\beta}{1-\beta^{n}} \sum_{r=1}^{n} \beta^{r-1} u\left(x_{[r]}\right)\right) .
\end{aligned}
$$

If $x_{[n]} \leq c$, then $e_{n+k}\left(\mathbf{x},(z)_{k}\right) \leq c$ for all $k \in \mathbb{N}$ and Step 1 is completed. Therefore, assume $x_{[n]}>c$, which, since $z \leq x_{[1]}$ and $z<c$, implies that $z<e_{n}(\mathbf{x})$.

Write $a_{k}:=\left(1-\beta^{k}\right) /\left(1-\beta^{n+k}\right)$; because $0<\beta<1,\left(a_{k}\right)_{k \in \mathbb{N}}$ is an increasing sequence converging to 1 . Since $z<e_{n}(\mathbf{x})$ and

$$
e_{n+k}\left(\mathbf{x},(z)_{k}\right)=u^{-1}\left(a_{k} u(z)+\left(1-a_{k}\right) u\left(e_{n}(\mathbf{x})\right)\right),
$$

it follows that $e_{n+k+1}\left(\mathbf{x},(z)_{k+1}\right)<e_{n+k}\left(\mathbf{x},(z)_{k}\right)$ and $e_{n+k}\left(\mathbf{x},(z)_{k}\right)$ tends to $z$ when $k$ tends to infinity. As $z<c$, we deduce that for any $n \in \mathbb{N}$ and $\mathbf{x} \in \mathbb{R}^{n}$, there exists $k(\mathbf{x}) \in \mathbb{N}$ such that for any $k \geq k(\mathbf{x}), e_{n+k}\left(\mathbf{x},(z)_{k}\right)<c$.

Step 2: For any $\mathbf{x}, \mathbf{y} \in \mathbf{X}$, choose $z$ with $z \leq \min \left\{x_{[1]}, y_{[1]}\right\}$ and $z<c, \ell=\max \{k(\mathbf{x}), k(\mathbf{y})\}$, $x=e_{n(\mathbf{x})+\ell}\left(\mathbf{x},(z)_{\ell}\right)$, and $y=e_{n(\mathbf{y})+\ell}\left(\mathbf{y},(z)_{\ell}\right)$, and use $\left(\mathbf{x},(z)_{\ell}\right) \sim(x)_{n(\mathbf{x})+\ell},\left(\mathbf{y},(z)_{\ell}\right) \sim$ $(y)_{n(\mathbf{y})+\ell}$ and $(x)_{n(\mathbf{x})+\ell},(y)_{n(\mathbf{y})+\ell} \in \mathbf{X}_{c}$ to establish the result. 
Using the above definitions of $x$ and $y$, we obtain the following equivalences by repeated applications of Axiom 5 and Lemma 2:

$$
\begin{aligned}
\mathbf{x} \succsim \mathbf{y} & \Leftrightarrow \quad(x)_{n(\mathbf{x})+\ell} \sim\left(\mathbf{x},(z)_{\ell}\right) \succsim\left(\mathbf{y},(z)_{\ell}\right) \sim(y)_{n(\mathbf{y})+\ell} \\
& \Leftrightarrow \quad \sum_{r=1}^{n(\mathbf{x})+\ell} \beta^{r}(u(x)-u(c)) \geq \sum_{r=1}^{n(\mathbf{y})+\ell} \beta^{r}(u(y)-u(c)) .
\end{aligned}
$$

However, by Lemma 1,

$$
\begin{aligned}
& \sum_{r=1}^{n(\mathbf{x})+\ell} \beta^{r} u(x)=\sum_{r=1}^{\ell} \beta^{r} u(z)+\beta^{\ell} \sum_{r=1}^{n(\mathbf{x})} \beta^{r} u\left(x_{[r]}\right) \\
& \sum_{r=1}^{n(\mathbf{y})+\ell} \beta^{r} u(y)=\sum_{r=1}^{\ell} \beta^{r} u(z)+\beta^{\ell} \sum_{r=1}^{n(\mathbf{y})} \beta^{r} u\left(y_{[r]}\right),
\end{aligned}
$$

using the fact $(x)_{n(\mathbf{x})+\ell} \sim\left(\mathbf{x},(z)_{\ell}\right)$ and $(y)_{n(\mathbf{y})+\ell} \sim\left(\mathbf{y},(z)_{\ell}\right)$. We obtain that

$$
\sum_{r=1}^{n(\mathbf{x})} \beta^{r}\left(u\left(x_{[r]}\right)-u(c)\right) \geq \sum_{r=1}^{n(\mathbf{y})} \beta^{r}\left(u\left(y_{[r]}\right)-u(c)\right)
$$

if and only if $\mathbf{x} \succsim \mathbf{y}$ by combining these result and rearranging terms.

Next, we provide a proof of Theorem 2.

Proof of Theorem 2. Assume that $\mathbf{x}, \mathbf{y} \in \mathbf{X}$, and $u$ is a continuous and increasing function.

Part (i). This part follows directly from the observation that on the domain $(0,1]$, $\sum_{r=1}^{n(\mathbf{x})} \beta^{r}\left(u\left(x_{[r]}\right)-u(c)\right)$ is a continuous function of $\beta$.

Part (ii). Since $\succsim_{c}^{L}$ is complete, it is sufficient to show that $\mathbf{x} \sim_{c}^{L} \mathbf{y}$ implies the existence of $\beta \in(0,1)$ such that $\mathbf{x} \sim_{\beta, u, c} \mathbf{y}$ for all $\beta \in(0, \beta)$, and that $\mathbf{x} \succ_{c}^{L} \mathbf{y}$ implies the existence of $\beta \in(0,1)$ such that $\mathbf{x} \succ_{\beta, u, c} \mathbf{y}$ for all $\beta \in(0, \bar{\beta})$.

$\mathbf{x} \sim_{c}^{L} \mathbf{y}$ implies the existence of $\beta \in(0,1)$ such that $\mathbf{x} \sim_{\beta, u, c} \mathbf{y}$ for all $\beta \in(0, \beta)$. Let $n(\mathbf{x}) \geq n(\mathbf{y})$. By Definition $5,\left(x_{[1]}, \ldots, x_{[n(\mathbf{y})]}\right)=\mathbf{y}_{[]}$and $\left(x_{[n(\mathbf{x})+1]}, \ldots, x_{[n(\mathbf{y})]}\right)=$ $(c)_{n(\mathbf{x})-n(\mathbf{y})}$. By Definition $1, \mathbf{x} \sim_{\beta, u, c} \mathbf{y}$ for all $\beta \in(0,1)$.

$\mathbf{x} \succ_{c}^{L} \mathbf{y}$ implies the existence of $\beta \in(0,1)$ such that $\mathbf{x} \succ_{\beta, u, c} \mathbf{y}$ for all $\beta \in(0, \beta)$. Let $x=\min \left\{x_{[1]}, c\right\}$ and $y=\max \left\{y_{[n(\mathbf{y})]}, c\right\}$. Note that $x \leq y$. If $x=y$ and $\mathbf{x} \succ_{c}^{L} \mathbf{y}$, then by Definitions 1 and $5, \mathbf{x} \succ_{\beta, u, c} \mathbf{y}$ for all $\beta \in(0,1)$. Hence, only the case where $x<y$ remains. By Definition 5, there are three cases. Case 1: There exists $R \in\{1, \ldots, n\}$ such that $x_{[r]}=y_{[r]}$ for all $r \in\{1, \ldots, R-1\}$ and $x_{[R]}>y_{[R]}$, where $n:=\min \{n(\mathbf{x}), n(\mathbf{y})\}$. In this case, let $x^{\prime}=x_{[R]}$ and $y^{\prime}=y_{[R]}$. Case 2: $n(\mathbf{x})>n(\mathbf{y}),\left(x_{[1]}, \ldots, x_{[n(\mathbf{y})]}\right)=\mathbf{y}_{[]}$, and $\left(x_{[n(\mathbf{y})+1]}, \ldots, x_{[n(\mathbf{x})]}\right)>(c)_{n(\mathbf{x})-n(\mathbf{y})}$. In this case, let $x^{\prime}=x_{[R]}$ and $y^{\prime}=c$, where $R:=\min \left\{r>n(\mathbf{y}): x_{[r]}>c\right\}$. Case 3: $n(\mathbf{x})<n(\mathbf{y}), \mathbf{x}_{[]}=\left(y_{[1]}, \ldots, y_{[n(\mathbf{x})]}\right)$, and $c>y_{[n(\mathbf{x})+1]}$. In this case, let $x^{\prime}=c$ and $y^{\prime}=y_{[n(\mathbf{x})+1]}$. Note that in all three cases, $x^{\prime}>y^{\prime}$. Define $\underline{\beta}$ by

$$
(1-\underline{\beta}) u\left(x^{\prime}\right)+\underline{\beta} u(x)=(1-\underline{\beta}) u\left(y^{\prime}\right)+\underline{\beta} u(y) .
$$


Then, by applying the affine transformation

$$
\frac{1-\beta}{\beta}\left(\sum_{r=1}^{n(\mathbf{x})} \beta^{r}\left(u\left(x_{[r]}\right)-u(c)\right)\right)+u(c)=(1-\beta) \sum_{r=1}^{n(\mathbf{x})} \beta^{r-1} u\left(x_{[r]}\right)+\beta^{n(\mathbf{x})} u(c),
$$

it follows from Definition 1 that $\mathbf{x} \succ_{\beta, u, c} \mathbf{y}$ for all $\beta \in(0, \underline{\beta})$.

Finally, we provide proofs of Propositions 1 and 2.

Proof of Proposition 1. Part (i). Consider an NDCLU SWO $\succsim$ such that $c>0$. Assume that $\succsim$ satisfies the Weak Non-Sadism Condition: there exist $y \in \mathbb{R}_{--}$and $k \in \mathbb{N}$ such that for all $\mathbf{x} \in \mathbf{X}, z \in \mathbb{R}_{++}$, and $n \in \mathbb{N},\left(\mathbf{x},(y)_{k}\right) \precsim\left(\mathbf{x},(z)_{n}\right)$. Consider any $v>c>z>0$. Choose $\ell \in \mathbb{N}$ such that ${ }^{6}$

$$
f(\ell+k)\left(\frac{\ell}{\ell+k}(u(v)-u(c))+\frac{k}{\ell+k}(u(y)-u(c))\right)>0 .
$$

It also possible to find $n \in \mathbb{N}$ such that ${ }^{7}$

$$
f(\ell+n)\left(\frac{\ell}{\ell+n}(u(v)-u(c))+\frac{n}{\ell+n}(u(z)-u(c))\right)<0 .
$$

By Definition 4, (A.3) and (A.4) imply that $\left((v)_{\ell},(y)_{k}\right) \succ\left((v)_{\ell},(z)_{n}\right)$, which is a contradiction.

Consider an NDCLU SWO $\succsim$ such that $c=0$. If the function $f$ is not bounded above, then for all $y>z>0$ and $k \in \mathbb{N}$, there is $n>k$ such that $f(k)(u(y)-u(0))<$ $f(n)(u(z)-u(0))$. Hence, by Definition $4,(y)_{k} \prec(z)_{n}$, which is the Repugnant Conclusion. Therefore, assume that the function $f$ is bounded above. Assume furthermore that $\succsim$ satisfies the Weak Non-Sadism Condition: there exist $y \in \mathbb{R}_{--}$and $k \in \mathbb{N}$ such that for all $\mathbf{x} \in \mathbf{X}, z \in \mathbb{R}_{++}$, and $n \in \mathbb{N},\left(\mathbf{x},(y)_{k}\right) \precsim\left(\mathbf{x},(z)_{n}\right)$. For $v>0$, choose like above $\ell \in \mathbb{N}$ such that

$$
f(\ell+k)\left(\frac{\ell}{\ell+k}(u(v)-u(0))+\frac{k}{\ell+k}(u(y)-u(0))\right)>0 .
$$

Denote the left-hand side by $M$. Because $f$ is bounded above and $u$ is continuous and increasing, there is $z>0$ such that $f(n)(u(z)-u(0))<M$ for all $n \in \mathbb{N}$. Hence, by the same reasoning as above, there exists $n \in \mathbb{N}$ such that

$$
f(\ell+n)\left(\frac{\ell}{\ell+n}(u(v)-u(0))+\frac{n}{\ell+n}(u(z)-u(0))\right)<M .
$$

By Definition 4, (A.5) and (A.6) imply that $\left((v)_{\ell},(y)_{k}\right) \succ\left((v)_{\ell},(z)_{n}\right)$, which is a contradiction.

\footnotetext{
${ }^{6}$ There necessarily exists such an $\ell \in \mathbb{N}$ because $f(\ell+k)>0$ and $(\ell /(\ell+k))(u(v)-u(c))+$ $(k /(\ell+k))(u(y)-u(c))$ is arbitrarily close to $u(v)-u(c)>0$ for large values of $\ell$.

${ }^{7}$ There necessarily exists such an $n \in \mathbb{N}$ because $f(\ell+n)>0$ and $(\ell /(\ell+n))(u(v)-u(c))+$ $(n /(\ell+n))(u(z)-u(c))$ is arbitrarily close to $u(z)-u(c)<0$ for large values of $n$.
} 
The arguments of part (i) hold even if the function $u$ is bounded above.

Part (ii). An RDCLU SWO $\succsim$ avoids the Weak Repugnant Conclusion. If $y>z>c$, then there exists $k \in \mathbb{N}$ determined by the requirement that

$$
\frac{1-\beta^{k}}{1-\beta}(u(y)-u(c)) \geq \frac{1}{1-\beta}(u(z)-u(c)),
$$

implying that $(y)_{k} \succ(z)_{n}$ for all $n \in \mathbb{N}$ according to the RDCLU SWO (cf. Definition 1). This holds even if the function $u$ is bounded above.

An RDCLU SWO $\succsim$ satisfies the Weak Non-Sadism Condition if the function $u$ is bounded above. Let $\bar{u}:=\sup \{u(x): x \in \mathbb{R}\}$, and choose $y \in \mathbb{R}_{--}$and $k \in \mathbb{N}$ such that

$$
\frac{1-\beta^{k}}{1-\beta}(u(y)-u(0))+\frac{\beta^{k}}{1-\beta}(\bar{u}-u(0)) \leq 0 .
$$

Then it follows from the fact that $\bar{u} \geq u(c) \geq u(0)$ that for any initial allocation $\mathbf{x} \in \mathbf{X}$ where all individuals have positive lifetime well-being, $\left(\mathbf{x},(y)_{k}\right) \prec\left(\mathbf{x},(z)_{n}\right)$ for all $z \in \mathbb{R}_{++}$and $n \in \mathbb{N}$. This obtains since (A.7) implies

$$
\frac{1-\beta^{k}}{1-\beta}(u(y)-u(c))+\frac{\beta^{k}}{1-\beta}(\bar{u}-u(c)) \leq \frac{1}{1-\beta}(u(0)-u(c)),
$$

where the r.h.s. is smaller than the welfare of any allocation where all individuals have positive well-being. Including individuals in $\mathbf{x}$ with well-being at or below $y$ does not change the ranking, while including individuals in $\mathbf{x}$ at $y$ and then raising their wellbeing toward 0 contributes less to $\left(\mathbf{x},(y)_{k}\right)$ than to $\left(\mathbf{x},(z)_{n}\right)$ since their rank is higher in the former allocation than in the latter. Hence, for any initial allocation $\mathbf{x} \in \mathbf{X},\left(\mathbf{x},(y)_{k}\right) \precsim$ $\left(\mathbf{x},(z)_{n}\right)$, showing that an RDCLU SWO satisfies the Weak Non-Sadism Condition if the function $u$ is bounded above.

Proof of Proposition 2. Part (i). Consider an NDCLU SWO $\succsim$ such that the function $f$ is bounded above. If, for some $n \in \mathbb{N}, f(n)>f(n+1)$, then Definition 4 implies that for any $z \in \mathbb{R}_{--},(z)_{n} \prec(z)_{n+1}$, so that the Negative Mere Addition Principle is not satisfied. Therefore, assume that $f$ is nondecreasing. Let $y<z<0$. If there exist $n \in \mathbb{N}$ and $k \in \mathbb{N}$ such that $\left((y)_{n},(z)_{k}\right) \succ(y)_{n}$, then by completeness and transitivity of an NDCLU SWO, there must exist $0 \leq \ell<k$ such that $\left((z)_{n},(y)_{\ell+1}\right) \succ\left((z)_{n},(y)_{\ell}\right)$, a violation of the Negative Mere Addition Principle. Hence, to establish a violation of the Negative Mere Addition Principle, it is sufficient to show that there exist $n \in \mathbb{N}$ and $k \in \mathbb{N}$ such that $\left((y)_{n},(z)_{k}\right) \succ(y)_{n}$ or, equivalently, by Definition 4 ,

$$
\frac{f(n)}{f(n+k)}>\frac{\frac{n}{n+k}(u(c)-u(y))+\frac{k}{n+k}(u(c)-u(z))}{u(c)-u(y)} .
$$

For sufficiently small $\varepsilon>0$, there is $n \in \mathbb{N}$ such that

$$
\frac{f(n)}{f(n+k)} \geq \frac{u(c)-u(z)}{u(c)-u(y)}+\varepsilon
$$


for all $k \in \mathbb{N}$, since any nondecreasing bounded sequence converges and $u(y)<u(z)<$ $u(c)$. The result follows since the numerator of the right-hand side of (A.8) is arbitrarily close to $u(c)-u(z)$ for large values of $k$.

Consider an NDCLU SWO $\succsim$ such that the function $f$ is not bounded above. If $c>0$, then for all $z \in \mathbb{R}_{++}$with $z<c, f(n)(u(z)-u(c))$ tends to minus infinity when $n$ goes to infinity. Therefore, by Definition 4 , for any $\mathbf{x} \in \mathbf{X}$ there exists $n \in \mathbb{N}$ such that $\mathbf{x} \succ(z)_{n}$, which is the Very Sadistic Conclusion. If, on the other hand, $c=0$, then for all $z \in \mathbb{R}_{++}$, $f(n) u(z)$ tends to infinity when $n$ goes to infinity. Hence, by Definition 4 , for all $y \in \mathbb{R}_{++}$ with $y>z>0$ and $k \in \mathbb{N}$, there is $n>k$ such that $(y)_{k} \prec(z)_{n}$, which is the Repugnant Conclusion.

Part (ii). An RDCLU SWO え avoids the Repugnant Conclusion. By Proposition 1(ii), any RDCLU SWO avoids the Weak Repugnant Conclusion and therefore avoids the Repugnant Conclusion.

An RDCLU SWO $\succsim$ avoids the Very Sadistic Conclusion. Choose $y \in \mathbb{R}_{--}$and $k \in \mathbb{N}$ such that $\left(1-\beta^{k}\right)(u(y)-u(c)) \leq u(0)-u(c) \leq\left(1-\beta^{n}\right)(u(0)-u(c))$ for all $n \in \mathbb{N}$. By Definition $1,(y)_{k} \precsim(0)_{n} \prec(z)_{n}$ for all $z \in \mathbb{R}_{++}$and $n \in \mathbb{N}$. Thus an RDCLU SWO avoids the Very Sadistic Conclusion.

An RDCLU SWO $\succsim$ satisfies the Negative Mere Addition Principle. This follows since $c$ is a lower bound for the context-dependent critical level for $\succsim$.

\section{REFERENCES}

Arrhenius, Gustaf (2000), Future Generations-A Challenge for Moral Theory. Ph.D. thesis, Uppsala University. [630, 636]

Arrhenius, Gustaf (forthcoming), Population Ethics. Oxford University Press, Oxford. [630, 631, 634, 636, 637, 638, 639]

Asheim, Geir B. (2010), “Intergenerational equity." Annual Review of Economics, 2, 197-222. [631]

Asheim, Geir B. and Stéphane Zuber (2014), "Probability adjusted rank-discounted utilitarianism.” Working Paper 4728, CESifo. [642]

Blackorby, Charles, Walter Bossert, and David Donaldson (1995), "Intertemporal population ethics: Critical-level utilitarian principles.” Econometrica, 63, 1303-1320. [633]

Blackorby, Charles, Walter Bossert, and David Donaldson (1996), "Leximin population ethics.” Mathematical Social Sciences, 31, 115-131. [630, 634, 637]

Blackorby, Charles, Walter Bossert, and David Donaldson (2002), "Population principles with number-dependent critical levels.” Journal of Public Economic Theory, 4, 347-368. [637]

Blackorby, Charles, Walter Bossert, and David Donaldson (2005), Population Issues in Social Choice Theory, Welfare Economics, and Ethics. Cambridge University Press, Cambridge. [630, 633, 634, 636, 637] 
Blackorby, Charles and David Donaldson (1984), "Social criteria for evaluating population change." Journal of Public Economics, 25, 13-33. [633]

Broome, John (2004), Weighing Lives. Oxford University Press, Oxford. [630]

Broome, John (2010), “The most important thing about climate change.” In Public Policy: Why Ethics Matters (Jonathan Boston, Andrew Bradstock, and David Eng, eds.), 101-116, ANU E Press, Canberra. [629]

Carlson, Erik (1998), "Mere addition and two trilemmas of population ethics." Economics and Philosophy, 14, 283-306. [639]

Dasgupta, Partha S. (1988), "Lives and well-being." Social Choice and Welfare, 5, 103-126. [639, 640]

Dasgupta, Partha S. (2005), “Regarding optimum population.” Journal of Political Philosophy, 13, 414-442. [640]

Ebert, Udo (1988), "Measurement of inequality: An attempt at unification and generalization.” Social Choice and Welfare, 5, 147-169. [642, 643]

Gorman, William M. (1968), "The structure of utility functions." Review of Economic Studies, 35, 367-390. [642, 643]

Ng, Yew-Kwang (1989), "What should we do about future generations? Impossibility of Parfit's theory X.” Economics and Philosophy, 5, 235-253. [631, 633]

Nordhaus, William D. (2008), A Question of Balance: Weighing the Options on Global Warming Policies. Yale University Press, New Haven, Connecticut. [630]

Palivos, Theodore and Chong K. Yip (1993), "Optimal population size and endogenous growth.” Economics Letters, 41, 107-110. [640]

Parfit, Derek (1976), “On doing the best for our children." In Ethics and Population (Michael D. Bayles, ed.), 100-102, Schenkman, Cambridge, Massachusetts. [630, 636]

Parfit, Derek (1982), "Future generations, further problems." Philosophy \& Public Affairs, 11, 113-172. [630, 636]

Parfit, Derek (1984), Reasons and Persons. Oxford University Press, Oxford. [630, 636]

Razin, Assaf and Chi-Wa Yuen (1995), "Utilitarian tradeoff between population growth and income growth.” Journal of Population Economics, 8, 81-87. [640]

Sider, Theodore (1991), "Might theory X be a theory of diminishing marginal value?" Analysis, 51, 265-271. [631]

Zuber, Stéphane and Geir B. Asheim (2012), "Justifying social discounting: The rankdiscounted utilitarian approach.” Journal of Economic Theory, 147, 1572-1601. [631, 639, 641]

Submitted 2012-9-13. Final version accepted 2013-6-3. Available online 2013-6-3. 